\title{
Table ronde 5
}

\section{Le médicament orphelin est-il en danger en tant que médicament développé dans les règles de l'art?}

\section{Antoine Ferry}

Cette table ronde risque d'être un peu agitée, car la répétition que nous avons faite pendant la pause-café a donné lieu à des échanges vifs.

Nous allons commencer par un panorama sur la vie du médicament, en pointant les éventuels dangers, qui ne sont pas là où l'on pense. Malheureusement pour les patients, un médicament ayant obtenu une AMM européenne ne sera pas forcément accessible dans les différents États européens. Le parcours jusqu'à I'AMM s'apparente à un vrai parcours du combattant. Après I'AMM, s'engage un autre parcours du combattant, qui ne semble pas s'améliorer au fil du temps.

Décomposé en quatre étapes, le schéma du danger a beaucoup évolué au cours de la décennie passée. II y a dix ans, j'aurais considéré que le danger devait être positionné de façon prépondérante dans les phases préclinique et clinique, et de façon mineure au niveau de l'enregistrement et de la commercialisation. Aujourd'hui, la situation s'est malheureusement inversée.

\section{Jean Pouget}

De vrais dangers menacent encore les étapes préclinique et clinique de l'essai d'un médicament. Ainsi, je considère qu'après une décennie, la situation ne s'est pas améliorée dans ces étapes précoces du développement du médicament.

\section{Serge Braun}

Les dangers sont tout aussi importants à chacune des quatre étapes.

\section{Christian Deleuze}

La phase préclinique démarre souvent à partir de rien. Les données physiopathologiques sont en effet peu nombreuses. L'identification de la cible présente une réelle difficulté. La validité et la prédictibilité des modèles in vitro sont faibles. De plus, les modèles animaux sont souvent inexistants. Des appels à projets pour des modèles animaux ont été lancés, notamment par la Fondation Maladies Rares. Nous espérons

Participent à la table ronde :

Serge Braun, AFM Téléthon

Antoine Ferry, Laboratoires CTRS

François Meyer, HAS

Jean Pouget, AP-HM

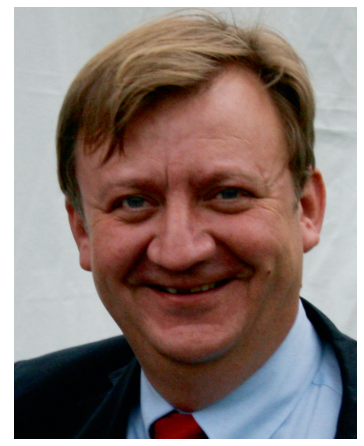

La table ronde est animée par Antoine Ferry

(Laboratoires CTRS)

qu'ils aboutiront pour pouvoir avancer plus rapidement sur la partie préclinique.

\section{Serge Braun}

J'estime que les dangers sont moindres à l'étape préclinique. L'origine de la pathologie rare est très souvent connue puisque $80 \%$ des maladies rares sont génétiques et plus de $50 \%$ de la moitié des gènes responsables sont connus. En outre, la physiopathologie de ces maladies est connue. C'est d'ailleurs une source très intéressante de connaissances fondamentales de la biologie, dans la mesure où un gène qui ne fonctionne pas renseigne sur la fonction de ce gène. La cible est parfois connue. Les modèles in vitro présentent des limites; il en va de même pour les modèles animaux. Pour les maladies rares, nous avons un avantage puisque le gène équivalent existe chez l'animal. II est donc possible d'accéder à une source très intéressante d'informations sur le plan physiopathologique et ensuite sur le plan thérapeutique.

\section{Jean Pouget}

Je ne suis pas d'accord. La plupart des maladies rares sont certes génétiques, mais, dans la majorité des cas, l'identification du gène ne suffit pas à comprendre la physiopathologie. La compréhension de la protéine mutée, découverte au travers du gène muté, n’est pas 
toujours implicite. L'anomalie génétique, n'impliquant pas forcément une protéine de structure ou une protéine enzymatique, intervient de manière complexe dans le fonctionnement cellulaire.

La construction de modèles animaux transgéniques constitue certes un progrès, mais ces modèles sont très souvent imparfaits. Ils nous ont beaucoup déçus dans la mesure où l'efficacité d'un certain nombre de traitements dans les modèles animaux ne s'est pas retrouvée chez l'homme. Le modèle animal est peut-être imparfait. II se peut également que le produit agisse à des moments différents de la maladie chez l'animal et chez l'homme.

Les modèles in vitro permettent d'aborder la physiopathologie, mais ils ne peuvent en aucun cas représenter un paramètre d'efficacité thérapeutique. Le modèle intégratif - basé sur l'animal entier - est toujours préféré au modèle cellulaire et au modèle in vitro. De nombreux progrès doivent encore être réalisés dans la physiopathologie, que ce soit dans les maladies auto-immunes rares ou dans les maladies neurodégénératives. Ce champ d'investigation demeurant totalement ouvert, il ne permet pas de répondre aux questions précliniques dans la majorité des maladies rares.

\section{Antoine Ferry}

La phase clinique est soumise à la rareté des pathologies. Le recrutement pour les études cliniques est difficile. L'expression clinique de ces pathologies présente une forte hétérogénéité. De plus, les données sur leur évolution naturelle sont généralement absentes. Par conséquent, la définition des critères de jugement (endpoints) pour les études cliniques est complexe ; le recours à des preuves indirectes de l'efficacité (surrogate endpoints) permet de quantifier l'efficacité d'un traitement, mais ces preuves sont rarement acceptées de facto par les autorités, notamment par l'EMA. Enfin, des problèmes de métabolisme différents selon les malades se posent. Le manque de prévisibilité peut poser des problèmes d'interprétation des résultats.

\section{Jean Pouget}

Le nombre de patients à inclure dans un essai thérapeutique présente des difficultés. Se pose également le problème de l'identification des patients pouvant être inclus. Le travail de constitution et d'actualisation des bases de données doit se poursuivre. Celles-ci doivent inclure des données phénotypiques. Des bases de données de qualité peuvent être utilisées à la fois pour la recherche clinique et les essais thérapeutiques. Cela pose la question de vouloir constituer un guichet unique pour les essais thérapeutiques au niveau européen. Certaines structures vont essayer de fusionner les bases de données nationales, qui présentent des niveaux de qualité différents et qui contiennent des données diverses. II faut donc trouver le bon interlocuteur, sans chercher à simplifier les choses via un guichet unique qui n'en est pas un. Ce dernier ne représente pas forcément la solution.

Le problème du critère principal d'évaluation est loin d'être résolu dans les maladies rares, car le consensus d'experts fait encore défaut. Les laboratoires et les agences se tournent vers les experts. Comme ils n'ont pas les bonnes réponses, l'essai sera bancal dès l'origine. Pour la maladie de Duchenne, le test de marche de six minutes ne correspond pas, de l'avis de l'ensemble des experts, à un bon paramètre pouvant servir de critère principal d'évaluation. II a pourtant été utilisé.

S'agissant des biomarqueurs, les agences ne vont pas se contenter d'une efficacité sur un biomarqueur, mais sur une amélioration fonctionnelle significative de la vie du patient. II serait peut-être judicieux de lancer des appels d'offres sur la méthodologie des essais dans les maladies rares.

\section{Serge Braun}

Les difficultés de recrutement sont bien sûr inhérentes aux maladies rares. Les règles de l'art de médicaments de thérapies fréquentes ne peuvent bien évidemment pas être appliquées aux maladies rares. Les premiers essais de thérapie génique menés au début des années 1990 sur les déficits immunitaires ont fait apparaître un taux de réussite de $90 \% .25$ ans plus tard, aucun produit n'a été mis sur le marché. Ce constat est-il lié à un problème d'évaluation, à un problème d'appréciation par les agences réglementaires, à des problèmes de production? La question continue de se poser.

Le test des six minutes est intéressant, mais il ne peut pas être appliqué aux patients qui ne marchent pas. Des paramètres de mobilité et de qualité de vie doivent donc être définis avec les agences, les associations de patients et les patients eux-mêmes.

S'agissant des surrogate endpoints, je souhaiterais partager une courte histoire. Un produit fait l'objet d'une demande d'AMM pour la myopathie de Duchenne. La FDA a rejeté l'utilisation du surrogate endpoint portant sur l'expression de dystrophine - c'est-à-dire la protéine manquante au niveau des muscles. Lorsque les données cliniques ont été transmises, la FDA a demandé que soit étudié le surrogate endpoint, alors que ce n'était pas prévu au départ. Cet exemple montre que la barre est placée très haute pour les maladies rares. Trois mois de survie dans le cancer s'apparentent à un grand succès ; il en irait tout autrement pour la maladie de Duchenne.

\section{François Meyer}

L'opposition entre les maladies n'est pas souhaitable. Le règlement sur le médicament orphelin a été construit après de nombreuses années de discussions. L'un de ses considérants est ainsi formulé : "Il importe que les patients souffrant de maladies rares aient droit à des médicaments dont la qualité, la sécurité et l'efficacité sont équivalentes à celles des médicaments dont bénéficient les autres patients; il y a donc lieu de soumettre les médicaments orphelins à la procédure d'évaluation habituelle. » II ne faut donc pas que les médicaments orphelins fassent l'objet d'une évaluation «au rabais ». 
Il existe bien évidemment des particularités dues à des difficultés méthodologiques liées aux faibles effectifs, à la mauvaise connaissance de l'histoire naturelle de la maladie, à la difficulté de se mettre d'accord sur des points de jugement clinique faisant consensus. Ces difficultés ne sont pas toutes spécifiques aux médicaments orphelins. Ainsi, les dispositifs médicaux visent souvent de petites populations.

Les agences réglementaires et d'évaluation ont compris que la difficulté de définir des points de jugement appelle à un dialogue précoce entre les développeurs de nouveaux médicaments et les autorités. Ce dialogue est mené depuis de nombreuses années avec les autorités d'enregistrement. Pour les médicaments orphelins, il s'agit de l'assistance protocolaire ou de l'avis scientifique. Depuis 2009, et surtout depuis 2012, les autorités d'évaluation pour le remboursement telles que la HAS se sont aussi engagées dans ce dialogue. L'identification du bon critère de jugement présente une réelle difficulté.

Force est de constater que pour les médicaments orphelins mis sur le marché, la qualité des données cliniques disponibles n'a pas toujours été optimale. À l'occasion du $10^{e}$ anniversaire du règlement médicament orphelin organisée à l'EMA, de nombreux cliniciens avaient expliqué et regretté que l'effet de certains médicaments orphelins sur l'évolution clinique des patients n'était toujours pas connu. II est de notre responsabilité collective de faire en sorte que ce problème ne se pose plus. Les agences d'évaluation prennent leur part sur ce sujet, à la fois par leurs initiatives personnelles et leur travail en commun.

Dans la définition des critères de jugement, des dialogues précoces par produit peuvent être conduits. En outre, l'EMA donne la possibilité de qualifier de nouveaux critères de jugement, lorsque les critères existants ne sont pas adaptés. Un suivi dans la vraie vie par des registres ou des cohortes permettra de confirmer que l'évolution clinique a été bénéfique.

La situation est encore plus complexe au niveau de l'évaluation en vue du remboursement. L'AMM quantifie le rapport bénéfice/risque, alors que les agences doivent évaluer le gain en santé. Ainsi, nous partageons totalement les difficultés des cliniciens dans ce domaine. Nous devons tous travailler ensemble pour essayer de définir les meilleurs critères - un vrai mouvement est en train de s'amorcer en ce sens.

\section{Antoine Ferry}

Nous poursuivons la présentation des problèmes rencontrés en clinique. La méthodologie statistique est spécifique aux faibles effectifs, ce qui pose un problème de recevabilité réglementaire.

Par ailleurs, il faut savoir que certains États-membres, au niveau des comités d'éthique, refusent les comparateurs (placebo, « gold standard » reconnu par l'agence, etc.).

Des produits de qualité pharmaceutique doivent être fabriqués pour mener des études cliniques. La production de ces petits lots constitue un vrai défi pour les industriels. Les sites disponibles étant peu nombreux à l'échelle européenne, les délais de production peuvent être relativement longs. De plus, les coûts de production sont élevés car les guidelines de fabrication des médicaments (GMP) ne sont pas adaptées au volume de production.

\section{Serge Braun}

Le placebo peut être nécessaire. II peut parfois être remplacé par les connaissances sur l'histoire naturelle de la maladie. Celle-ci suppose l'allocation de moyens très importants pour créer des bases de données de malades. Pour beaucoup de pathologies dans lesquelles nous sommes directement impliqués, nous constatons que les moyens financiers nécessaires reposent sur les associations. Ce problème de santé publique devrait être posé.

\section{François Meyer}

Le grand succès du règlement européen sur les médicaments orphelins est que ces médicaments ne sont plus tout à fait orphelins. L'arrivée de grands laboratoires sur ce marché marque une réelle évolution. Des laboratoires viennent parfois nous voir en nous expliquant que la première étude qu'ils entendent mettre en place porte sur l'histoire naturelle de la maladie. Le financement n'est donc pas exclusivement associatif. Dans certains cas, les laboratoires comprennent la nécessité de mettre en place ce type d'études. Tout n'est pas pour autant réglé. De grands besoins de financement demeurent.

\section{Jean Pouget}

Je ne suis pas sûr que les cohortes historiques puissent remplacer le placebo parce que le médicament ne constitue pas le seul élément des prises en charge. La qualité de ces dernières s'est grandement améliorée. Nous disposons de multiples preuves pour montrer que la prise en charge des conséquences de la maladie a significativement influé sur la fonction et la survie. II convient donc de comparer les choses à qualité de prise en charge équivalente, ce qui peut poser problème dans les essais internationaux. Des référentiels de prise en charge existent, mais ils sont probablement appliqués de manière disparate dans les différents pays.

Dans l'évolution naturelle, il convient de tenir compte de l'aspect temporel. Plus l'apparition de la maladie est précoce, plus la maladie sera évolutive. Pour des maladies extrêmement chroniques, la durée d'un essai thérapeutique (d'un à deux ans) ne peut pas rendre compte d'une aggravation «mesurable » dans l'évolution naturelle de la maladie. Si l'essai doit durer plusieurs années, des problèmes de coût et de réalisation se poseront. En outre, l'évaluation des maladies d'évolution lente est plus difficile.

\section{Antoine Ferry}

Dans la phase d'enregistrement, se posent des difficultés d'évaluation de la qualité pharmaceutique et 
de l'efficience de certaines thérapies innovantes. Des efforts doivent encore être faits sur l'appréciation de ces produits.

Un problème réglementaire se pose avec les Plans d'Investigation Pédiatrique (PIP) en concordance avec le calendrier général de l'évaluation du dossier d'enregistrement. Sauf dérogation, le PIP est obligatoire avant I'AMM. Le calendrier du CHMP et le calendrier du Comité Pédiatrique se chevauchent, ce qui provoque parfois des situations de blocage très préjudiciables.

Par ailleurs, certaines obligations relatives aux Bonnes Pratiques de Fabrication (BPF) ont un impact financier parfois considérable. Les mêmes règles de production doivent être appliquées. Même si le laboratoire ne produit que quatre lots, il doit chaque année en mettre un en stabilité pour avoir les données de stabilité. In fine, les coûts élevés des lots de ces petites séries doivent être amortis sur le prix du produit.

\section{François Meyer}

Le règlement pédiatrique est issu de la même philosophie que le règlement orphelin. Les avantages sont compensés par des obligations. Je comprends les difficultés de faire une concordance. Je m'étonne que le Comité pour l'évaluation des risques en matière de pharmacovigilance (PRAC) de l'EMA ne soit pas mentionné sur ce transparent. II aura peut-être des choses importantes à dire sur les médicaments orphelins. Issu de l'ancien groupe de travail de pharmacovigilance, le PRAC est devenu un comité à part entière. II pourra formuler des demandes d'études supplémentaires après l'AMM, non seulement sur la sécurité, mais aussi sur l'efficacité. Le paysage réglementaire européen s'est considérablement enrichi ; les acteurs sont multiples et leurs interactions nombreuses. Ainsi, je comprends les difficultés que cela peut générer.

L'évaluation de l'efficience est nécessaire et bénéfique ; il est normal que la société sache si aux fonds investis dans le domaine de la santé correspond bien un progrès thérapeutique en rapport avec l'effort financier consenti. II est logique que les citoyens s'assurent que l'argent investi a permis d'obtenir des données probantes et un progrès réel.

S'agissant de la qualité et de l'évaluation clinique, nous essayons d'améliorer les choses avec les dialogues précoces et les avis scientifiques. Beaucoup d'expérience doit encore être accumulée dans ce domaine.

Le concept de l'arrivée en deux temps des nouveaux médicaments (appelés Adaptive pathways) pourrait s'appliquer plus particulièrement aux médicaments orphelins.

\section{Serge Braun}

S'agissant des aspects qualité et efficience des thérapies innovantes, nous apprenons en marchant. Dans le secteur des biotechnologies, les standards sont parfois définis avec les inspecteurs parce qu'ils apprennent eux-mêmes de ces technologies. Le dialogue s'est instauré, et doit se poursuivre à tous les niveaux, y compris dans la négociation des prix. Les interactions entre les acteurs sont primordiales à tous les niveaux du processus de développement. L'industrie pharmaceutique commence à s'intéresser aux maladies rares - cette évolution est à saluer. Le marché visé doit cependant présenter une taille minimale pour permettre à la fois le retour sur investissement et la déclinaison des technologies développées sur des pathologies plus fréquentes.

Nous sommes aujourd'hui à la croisée des chemins, ce qui explique l'enthousiasme de nos discussions. L'arrivée de nombreuses thérapies innovantes dans des domaines très variés est une vraie bonne nouvelle. Cette évolution conduira à nous interroger sur le poids socio-économique de ces thérapies coûteuses qui seront appliquées à un nombre croissant de malades et de pathologies.

\section{Antoine Ferry}

Après l'obtention de l'AMM européenne, la phase de commercialisation peut débuter. L'Europe se caractérise par une forte hétérogénéité d'accès aux traitements et aux marchés. Si l'accès au marché est immédiat en Allemagne, l'Italie fait preuve d'une grande lenteur dans ce domaine. En outre, certains pays imposent de mener des renégociations de prix au niveau régional. Les obligations d'études post-AMM et d'études médicoéconomiques impactent également la durée avant la commercialisation.

La directive stipule que le médicament orphelin, en tant que médicament à part entière, doit répondre aux mêmes critères post-AMM que les autres médicaments. En outre tous les États-membres doivent se doter d'une structure nationale de pharmacovigilance et d'une structure d'information médicale, et respecter de nombreuses contingences réglementaires, même si la pathologie concerne moins d'une trentaine de personnes en Europe réparties entre cinq à six États-membres. Une concertation devrait être menée avec les autorités de régulation car l'impact financier de ces dispositions est considérable pour les médicaments ultra-orphelins traitant 20 à 40 patients. À titre d'exemple, le coût de ces contingences en Lituanie s'élève à 14000 euros alors que le pays ne compte aucun malade!

S'agissant des systèmes de fixation de prix et de capping sur des données de population incertaines, force est d'admettre que l'utilisation des registres et des cohortes permettrait de disposer de données communes fiables sur lesquelles les industriels et les autorités de régulation pourraient statuer.

Par ailleurs, il faut savoir que certains pays ne respectent pas la directive européenne en continuant à réaliser des préparations hospitalières, vendues à des prix ridiculement bas. D'un côté, la directive impose le respect d'impératifs de sécurité, de surveillance; de 
l'autre, les pharmacies hospitalières ne sont tenues à aucun impératif. Je ne critique pas pour autant les pharmacies hospitalières. En France, elles s'abstiennent de produire des préparations hospitalières dès lors qu'un médicament est disponible sur le territoire national.

Une étude menée aux États-Unis a fait couler beaucoup d'encre sur une contamination provoquant des méningites fongiques. Plus de 700 patients ont été contaminés et 60 personnes sont décédées à cause d'une préparation hospitalière. Quelques études prouvent la variabilité des teneurs de ces préparations. Si un industriel venait à présenter une variabilité du principe actif comprise entre 67 et $268 \%$, l'EMA serait peu encline à lui accorder un avis positif.

Lorsqu'un traitement a été évalué et parfaitement quantifié, la directive doit être respectée. Tous les États-membres n'ont pas encore fait le choix d'interdire les préparations hospitalières dès lors qu'un médicament est disponible sur leur territoire national.

\section{Jean Pouget}

En France, en maladie de la jonction neuromusculaire, un produit fabriqué par la pharmacie de l'AP-HP est passé en fabrication industrielle, entraînant une hausse de prix de 20 centimes à 20 euros. Ce facteur de correction est-il justifié par les obligations de la fabrication industrielle?

\section{Antoine Ferry}

Une succession d'obligations doivent être respectées en production et après I'AMM. L'impact financier de ces obligations, divisé par un petit nombre de patients, explique l'augmentation considérable du prix du médicament. Une simple règle de trois s'applique. En rapportant 2 millions d'euros de dépenses fixes (c'est-à-dire réglementaires) d'un médicament rapportés à une vingtaine de patients, vous obtenez l'impact du coût de traitement de ces obligations réglementaires. La pharmacie hospitalière ne connaît pas cette problématique, parce qu'elle n'est pas contrôlée de la même façon sur le principe actif et sur la qualité de la production; en outre, elle n'est pas tenue de respecter des obligations en pharmacovigilance.

\section{Jean Pouget}

Sur un plan de politique financière, ne vaudrait-il pas mieux améliorer la qualité de fabrication des pharmacies hospitalières plutôt que de confier la production de médicaments à un industriel ?

\section{François Meyer}

Le règlement est européen. Si une $A M M$ est demandée, le règlement doit s'appliquer. II y a parfois des réactions très fortes à des différences de prix paraissant - à juste titre ou non - excessives. S'il y a des mesures mises en place par certains pays qui ne sont pas conformes au règlement, ces mesures doivent être supprimées. Comme nous sommes dans un marché unique, des importations de produits à partir d'un pays où il serait vendu à un prix assez bas vers un pays à prix élevé sont possibles. Ceci est une limite à l'indépendance de la fixation des prix par chaque État-membre. Jusqu'à présent, les collaborations européennes entre décideurs en termes de prix et de remboursement ont été très peu nombreuses. Une réflexion a débuté sur ce sujet, en veillant à ce que chaque État-membre conserve la liberté de fixer son propre prix et visant à permettre la mise à disposition du médicament dans l'ensemble des pays. Cette réflexion a été accélérée par l'exemple des nouveaux médicaments de l'hépatite $C$.

\section{Antoine Ferry}

Des obligations réglementaires excessives pourraient générer les risques suivants:

- un frein important au développement pour les équipes de recherche;

- un désinvestissement des industriels, notamment sur les projets de repositionnement ou hors brevet ;

- une surenchère des coûts réglementaires non amortissables pour les industriels.

La diminution des investissements représenterait une perte de chance pour les malades et leurs familles. Trois propositions mériteraient d'être étudiées :

- le renforcement des recherches épidémiologiques;

- I'introduction d'une certaine flexibilité réglementaire tant en pré-AMM qu'en post-AMM ;

- la sécurisation du marché par le respect du cadre juridico-réglementaire et de la jurisprudence. $\diamond$

\section{ÉCHANGES AVEC LA SALLE}

\section{De la salle}

Pourquoi un traitement dont les études cliniques ont démontré sans équivoque l'efficacité est-il limité en quantité de remboursement, entraînant 50 \% de la facture payée par les laboratoires pharmaceutiques, et mettant les professionnels de santé en difficulté vis-àvis de leurs «fournisseurs » qui pourraient à l'avenir se retirer du marché français?

\section{Antoine Ferry}

La notion de capping sous-tend cette question. Le capping du nombre de patients traités par un industriel existe dans de nombreux pays européens ; ce n'est pas une particularité de la France.

\section{Christian Deleuze}

Les autorités de santé en France raisonnent en enveloppe fermée via la loi de financement de la Sécurité sociale. Chaque année, le coût global de tous les médicaments en France doit baisser de 1 à $2 \%$. Si cette baisse n'est pas respectée, un système de remboursement par les laboratoires est organisé. 


\section{François Meyer}

Si la population cible d'un médicament a été sous-estimée, une réévaluation reste possible. Le risque de retrait du marché français est donc limité.

\section{Antoine Ferry}

Le retrait du marché français serait la politique de l'industriel. II s'agit seulement d'une menace.

\section{Jean Pouget}

Le même système d'enveloppe finie existe aussi pour la tarification à l'activité.

\section{De la salle}

Pouvez-vous nous en dire un peu plus sur les qualification meetings pour validation des critères cliniques?

\section{François Meyer}

Les questions posées à l'EMA dans ce cadre (qualification d'un nouveau critère de jugement ou d'une nouvelle approche méthodologique pour le développement d'un médicament) sont actuellement faites par une seule firme, dans le cadre du développement clinique d'un médicament donné. Nous faisons pression pour que cette disposition puisse être ouverte à plusieurs laboratoires et que ses résultats ne soient pas confidentiels. En pratique, imaginons que pour une affection donnée deux nouveaux traitements sont en cours d'essai, et que l'utilisation de nouveaux critères d'évaluation, non encore validée, semble nécessaire. Un nouveau critère d'évaluation ou une nouvelle méthode d'évaluation peut être proposée à l'EMA, et probablement demain également aux agences d'évaluation en vue du remboursement.

\section{De la salle}

La FDA et l'EMA peuvent-ils avoir des avis discordants?

\section{François Meyer}

Des discordances sont possibles, mais des réunions communes sont organisées pour échanger.

\section{De la salle}

Comment expliquer que différentes agences aboutissent à des conclusions différentes, alors que le dossier est le même?

\section{Antoine Ferry}

Différentes modalités d'évaluation - et non différentes agences - peuvent aboutir à des conclusions différentes. En France, la HAS via la Commission de transparence adresse son évaluation au CEPS. Dans d'autres pays, les commissions sont mixtes. Certains pays disposent même de trois entités, en fonction du type de produit et de son évolution sur le marché. Les systèmes d'évaluation s'appuient sur des critères similaires, mais l'impact économique intervient parfois beaucoup plus tôt dans l'évaluation de l'« efficience ».

\section{François Meyer}

Il est normal que différentes agences aboutissent à des conclusions différentes sur un même dossier. II en a toujours été ainsi, car tout jugement revêt une dimension humaine. Nous sommes aujourd'hui habitués aux AMM centralisées. Pour rendre une seule conclusion, il faut tous se réunir dans une même pièce. Lors des rencontres précoces avec les industriels, nous observons de plus en plus que nos convergences sont beaucoup plus importantes que nos divergences.

\section{De la salle}

La HAS serait-elle prête à envisager des programmes de risque avec les industriels?

\section{Antoine Ferry}

Les risques financiers seraient pris par le CEPS, et non par la HAS. Cette question est liée à la problématique du contrat de performance. À titre personnel, je pense que nous devrions travailler sur cet impératif. RARE 2017 sera sans doute l'occasion d'échanger sur ce sujet. $\diamond$

\section{LIENS D'INTÉRÊT}

A. Ferry déclare avoir une participation financière dans le capital de l'entreprise Laboratoire CTRS et déclare avoir des liens durables avec l'entreprise Laboratoire CTRS.

C. Deleuze déclare avoir des liens durables avec l'entreprise SanofiGenzyme.

F. Meyer, S. Braun, J. Pouget déclarent n'avoir aucun lien d'intérêt concernant les données publiées dans cet article. 